\title{
Chronic Suppurative Osteomyelitis of the Maxilla - rare case presentation
}

SADJ April 2019, Vol. 74 No. 3 p 126 - p128

S Karpagavalli', VK Vaishnavi Vedam², G Sivadas ${ }^{3}$, S Sathish ${ }^{4}$, VN Kalaivani ${ }^{5}$, S Sushmita Naidu ${ }^{6}$

\begin{abstract}
Chronic Suppurative Osteomyelitis of the maxilla is a relatively uncommon lesion with scarce information in the medical literature regarding its clinical/pathological status and treatment perspectives.
\end{abstract}

We report a rare clinical case of a patient presenting with osteomyelitis of the maxilla with pus discharge. The review discusses the pathogenesis, clinical course, investigative procedures and treatment. The diagnosis in this patient was based on data assessed from a specialized imaging technique and from the histopathological findings. Timely medical and surgical therapy reduced the morbidity which may otherwise have occurred due to the significant complications associated with this chronic lesion. This interventional approach can thus be recommended as one of the novel treatments for osteomyelitis of the jaws with the prospect of significant clinical and radiological improvement, as was shown in the present case.

\section{Keywords}

Osteomyelitis, chronic suppurative osteomyelitis, maxillary osteomyelitis.

\section{Author affiliations:}

1. Shanmugasundaram Karpagavalli: MDS, Professor, Department of Oral Medicine and Radiology, Saveetha Dental College, Saveetha University, Chennai, Tamil Nadu, India.

2. Venkata Kanthi Vaishnavi Vedam: MDS, Senior Lecturer, Department of Oral Pathology, Faculty of Dentistry, Asian Institute of Medicine, Science \& Technology (AIMST) University, Semeling, Kedah, Malaysia.

3. Ganapathy Sivadas: MDS, Senior Lecturer, Department of Paedodontics and Preventive Dentistry, Faculty of Dentistry, Asian Institute of Medicine, Science \& Technology (AIMST) University, Semeling, Kedah, Malaysia.

4. Sivan Sathish: MDS, Reader, Dept. of Oral Medicine \& Radiology, Chettinad Dental College \& Research Institute, Kancheepuram, Tamil Nadu, India.

5. VN Kalaivani: BDS, Graduate, Chettinad Dental College \& Research Institute, Kancheepuram, Tamil Nadu, India.

6. Songa Sushmita Naidu: BDS, Graduate, Chettinad Dental College \& Research Institute, Kancheepuram, Tamil Nadu, India.

Corresponding author: Venkata Kanthi Vaishnavi Vedam

Department of Oral Pathology, Faculty of Dentistry, Asian Institute of Medicine, Science \& Technology (AIMST) University, Semeling, Kedah, Malaysia 08100.

Tel: +6017 6447178 Email: vaishnavivedam@gmail.com Author contributions:

1. Shanmugasundaram Karpagavalli: Principal researcher

2. Venkata Kanthi Vaishnavi Vedam: Manuscript preparation - $30 \%$

3. Ganapathy Sivadas: $25 \%$

4. Sivan Sathish: $20 \%$

5. VN Kalaivani: $15 \%$

6. Songa Sushmita Naidu: $10 \%$

\section{ACRONYM}

OPG: Orthopantamogram

\section{INTRODUCTION}

Osteomyelitis of the jaws is a lesion with distinct features, but is often challenging in diagnosis and treatment. It is an inflammatory condition involving the medullary portion of the bone, the Haversian system of the cortex and the periosteum, ${ }^{1}$ and was originally described by Rees in $1947 .{ }^{2}$ Even though medical and surgical management have advanced, the best prognosis is secured when treatment can be delivered at the initial stage of the disease. The incidence of maxillary osteomyelitis is low as the bone has thin cortical plates associated with a high degree of vascularity. We report a case of an adult patient presenting chronic suppurative osteomyelitis at the uncommon site of the maxilla, having an unusual etio-pathogenesis but with typical manifestation. Treatment was rendered in the classic mode. A review of the current pertinent literature is included.

\section{CASE REPORT}

A 52 year old female patient came to the Department of Oral Medicine and Radiology with the main complaint of pain accompanied by a purulent malodorous pus discharge from the posterior area of the maxilla on the right side, extending over the past three months. The patient had experienced a gradual onset of swelling and pain in that area and in the midfacial region, commencing some six months previously. The medical history included reports of recurrent asthmatic episodes controlled by medication for three years. Two years ago, the patient had undergone extraction of a decayed tooth on the upper left side, following which, three months later, swelling and pus discharge had developed at the extraction site, necessitating surgical management. Personal and family histories were non-contributory.

On extra-oral examination, facial asymmetry was observed with diffuse swelling evident on the right side of the mid-facial region, obliterating the nasolabial fold. On palpation, the swelling was tender, soft in consistency and fluctuant. There was no evidence of paresthesia. A single submandibular lymph-node could be felt on the right side, being tender, soft and freely mobile, and measuring about $1 \mathrm{~cm}$ in size. 


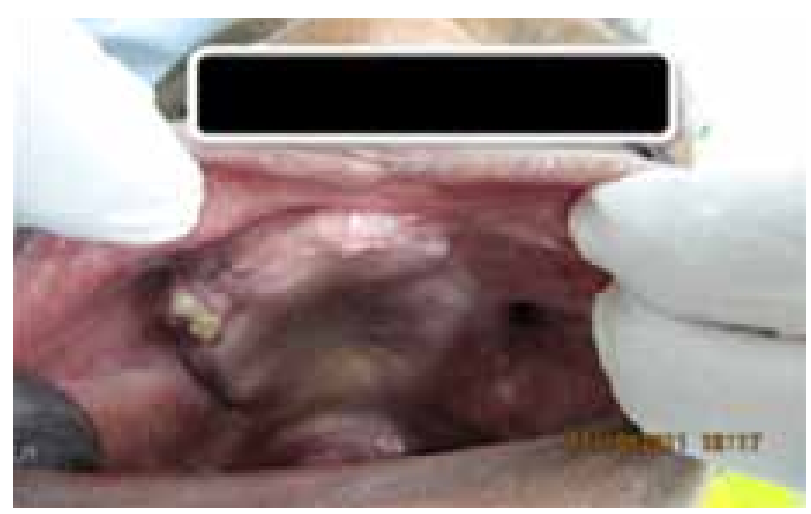

Figure 1. Intra-oral picture showing swelling with pus discharge and bony sequestra in relation to the right maxillary edentulous posterior region and a left sided side oro-antral fistula.

On intra-oral examination, a single localized swelling, with discharging pus, was evident. It extended from the edentulous region of right maxillary permanent canine to the second molar region (Figure 1). The swelling had obliterated the adjacent labial and buccal sulci, was soft in consistency and fluctuant on palpation, with a contained bony sequestrum.

On the left side, an oro-antral fistula was discovered.

An orthopantamogram (OPG) revealed an extensively destructive lesion with bony sequestra of the right maxilla and an oro-antral communication on the left maxilla.

The radiograph also confirmed multiple missing maxillary and posterior mandibular teeth (Figure 2a). An intraoral

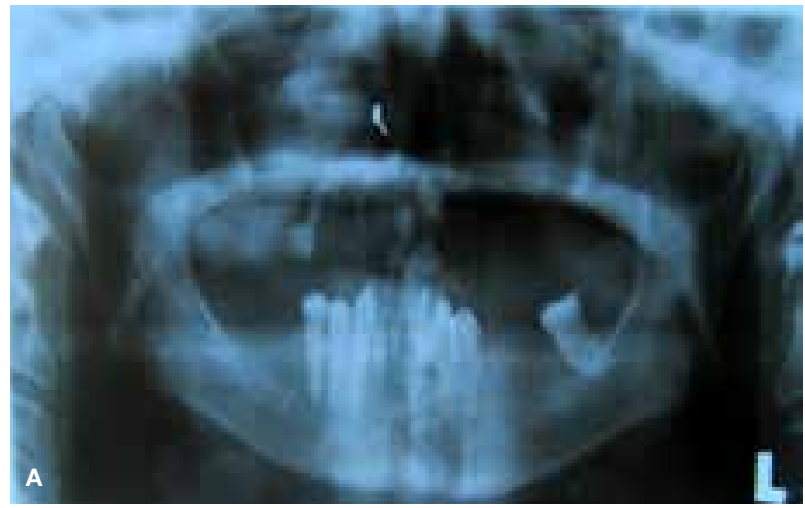

Figure $\mathbf{2 a}$ and $\mathbf{2 b}$. Orthopantamogram showing extensive destructive lesion of right maxilla with bony sequestra and an oro-antral communication in the left maxilla with multiple missing maxillary and mandibular teeth.

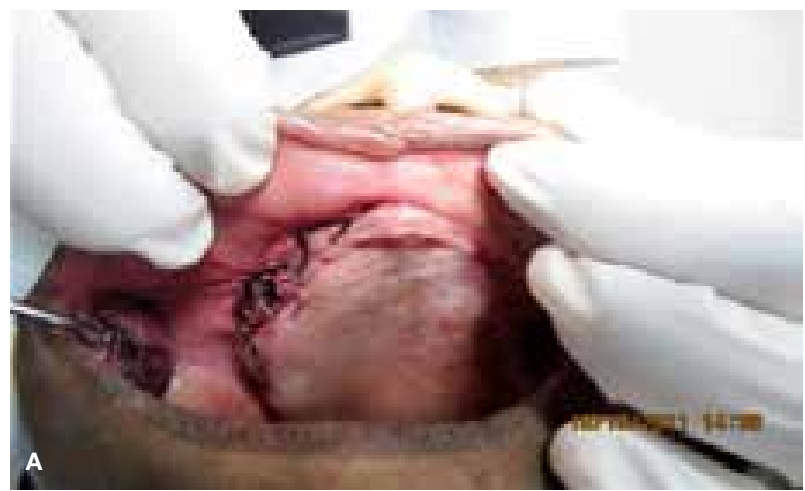

periapical radiograph of the right sided lesion revealed scattered areas of lytic changes with bony islands, along with sequestra. A provisional diagnosis of right maxillary osteomyelitis was made.

Subsequently, the wound was treated by surgical curettage, debridement and bone sequestrectomy. Intraoral suturing was performed after excision of the sinus tract.

Antibiotics were prescribed for the patient, taking into account her physical and medical health status.

The surgical specimen was placed in 10\% neutral formalin and sent for routine histopathological examination, which revealed a basophilic necrotized bone tissue with foci of calcified material surrounded by inflammatory cells and haemorrhage (Figure 2b).

The history, clinical picture and investigations confirmed a final diagnosis of chronic suppurative osteomyelitis of the right maxilla. The patient was followed up regularly fortnightly for next three months and has been in a disease free state over the past two years. (Figures $3 a$ and $3 b$ )

\section{DISCUSSION}

Osteomyelitis refers to inflammation of bone and its marrow contents. In Greek mythology, "osteon" means bone and "muelinos" means the medullary portion of bone. This lesion is of utmost importance as neglect can lead to various serious conditions like cavernous sinus thrombosis, meningitis, cerebral abscess and septicaemia. ${ }^{3}$

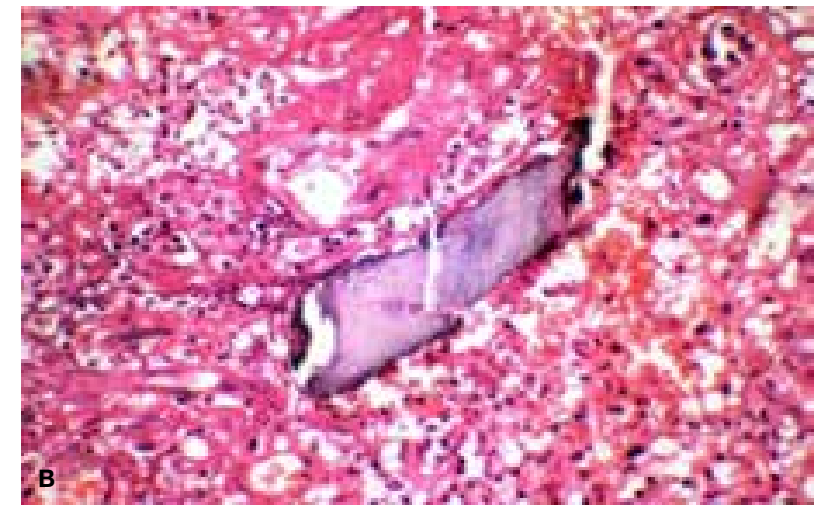

Histopathological picture (40x magnification) exhibiting necrotized bone associated with surrounding inflammation and haemorrhage.

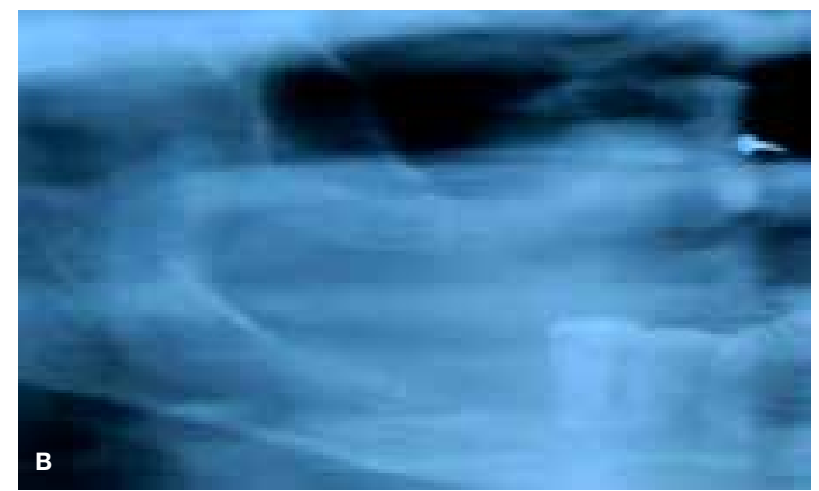

Figure 3a and 3b. Post-operative photograph showing the sutured right maxillary posterior region and a post-operative radiograph showing no evidence of dead bone, with healing in the right maxillary right posterior region following the surgical procedure. 
Waldegrel and Lew classified osteomyelitis of jaw bones broadly as "Suppurative or Non-suppurative". Clinically, it is further divided as:

A. Acute suppurative

B. Subacute

C. Primary chronic (no acute phase)

D. Non-suppurative

The occurrence of osteomyelitis in the jaws is rare and multi-factorial. Mandibular osteomyelitis occurs 3\%-19\% more frequently than does maxillary osteomyelitis.

The following factors may contribute to the occurrence of maxillary osteomyelitis:

A. Traumatic (teeth/lacrimal apparatus/antrum injuries/ compound fracture/gingival ulceration - furuncle/periosteitis)

B. Odontogenic (decayed tooth/primary tooth germ)

C. Surgical site/open soft tissue wound

D. Polymicrobial infection - diabetes, malignancy, malnutrition, bone metabolic diseases and immunosuppression altering host response

E. Osteoradionecrosis involving chronic infections (radiation therapy).

Although the most common site of chronic suppurative osteomyelitis is antral or in the odontogenic apparatus, our case could be attributed either to idiopathic/generalized immunosuppression or to pre-existing bacteria present in the dental biofilm of gingivitis and periodontitis, which may be the source of infection if other etiologies are excluded.

The prognosis of patients with osteomyelitis depends on several factors such as the characteristics and virulence of the microbial agents (staphylococcus aureus and staphylococcus epidermitidis), ${ }^{4}$ host immune response, source of infection and local tissue perfusion. ${ }^{5}$ The pathophysiology involves the accumulation of inflammatory exudates due to microbial infection in the bony cavities resulting in compression of the central and peripheral blood supply to bone. Reduction in oxygen supply and nutrients occur due to a compromised blood supply. ${ }^{6}$

Osteomyelitis occurs more commonly in males between fifty to sixty years of age. ${ }^{7}$ This lesion is usually seen in the posterior region of mandible where the cortical plates are thick and there is limited blood supply. Patients exhibit swelling, pain, pus discharge, paresthesia, trismus and regional lymphadenopathy. Pathological fractures and paresthesia are common complications of osteomyelitis. Progressive bone destruction and the presence of sequestra are traits of osteomyelitis. ${ }^{8}$

The present case occurred in a female patient in the relatively rare site of the posterior maxilla, but with typical symptoms. This lesion presented as extensively destructive, involving a diffuse radiolucency mixed with radiopaque calcification suggestive of a 'Moth eaten' appearance. Histopathologically, the tissue exhibited foci of calcification and necrosis of bone tissue with sequestra surrounded by mixed inflammatory cells (PMNs, lymphocytes and plasma cells). Osteoblasts were absent in the tissue specimen, suggesting a deficiency of new bone formation. ${ }^{9}$
Medications in the case included steroids, antibiotics and bisphosphonate therapy. Surgical management options included saucerisation, sequestrectomy, debridement, decortications, jaw resection with reconstruction, and hyperbaric oxygen. The case under report was surgically debrided, curettaged and sequestered. ${ }^{10}$

Correct diagnosis of the rarely seen chronic suppurative osteomyelitis of maxilla, with early intervention including a less invasive procedure, saved this patient from significant morbidity.

\section{Financial support}

None.

\section{Conflict of interest}

None.

\section{References}

1. Manimaran K, Suresh Kannan P, Kannan R. Osteomyelitis of maxillae -bilateral involvement: a case report. JIADS 2011; 2 (2): $57-8$

2. Vora M, Suryavanshi H, Baonerkar H. Surgical management of chronic suppurative osteomyelitis of mandible in elderly patient - a case report. International Journal of Current. Research and Review. 2014; 6(9): 58-64.

3. Wolfowitz B. Osteomyelitis of maxilla. South African Medical Journal 1971: 632-3.

4. Elerson GJ Jnr, Fardin AC, Jardim ECG. Microbiota associated with chronic osteomyelitis of jaws. Brazilian Journal of Microbiology 2010; 41: 1056-64.

5. Mallikarjun K, Kohli A, Kumar A, Tanwar A. Chronic suppurative osteomyelitis of mandible. Journal of Indian Society of Pedodontics and Preventive Dentistry 2011; 2(29): 176-9.

6. Rohit S, Roopak B, Prakash S. Condylar osteomyelitis secondary to dental extraction : an unforeseen consequence. International Journal of Clinical Dentistry 2012; 3(2): 85-8.

7. Shenoi R, Kolte V, Badjate S, Devrukhar V. Recurrent chronic suppurative osteomyelitis: review of literature and report of case. Indian Journal Interdisciplinary Dentistry 2010; 1(1): 29-32.

8. Ali K, Akram A, Akhtar MU. An unusual case of chronic suppurative osteomyelitis of mandible. Archives of Orofacial Sciences 2012; 7(1): 37-41.

9. Mudhol A, Ali FM, Barde D, Prashant MC. Chronic suppurative osteomyelitis of the mandible: case report and review. Scholars Journal of Medical Case Reports 2014; 2(6): 356-9.

10. Singh M, Singh S, Jain J. Chronic osteomyelitis of maxilla mimicking actinomycotic osteomyelitis: a rare case report. National Journal of Maxillofacial Surgery 2010; 1(2): 153. 